\title{
On Competing Mechanisms under Exclusive Competition*
}

\author{
Andrea Attar ${ }^{\dagger}$ Eloisa Campioni ${ }^{\ddagger}$ Gwenaël Piaser ${ }^{\S}$
}

May 23, 2018

\begin{abstract}
We study games in which several principals design mechanisms in the presence of privately informed agents. Competition is exclusive: each type of each agent can participate with at most one principal and meaningfully communicate only with him. Exclusive competition is at the centre stage of recent analyses of markets with private information. Economic models of exclusive competition restrict principals to use standard direct mechanisms, which induce truthful revelation of agents' exogenous private information. This paper investigates the rationale for this restriction. We provide two results. First, we construct an economic example showing that direct mechanisms fail to completely characterize equilibrium outcomes even if we restrict to pure strategy equilibria. Second, we show that truth-telling strongly robust equilibrium outcomes survive against principals' unilateral deviations toward arbitrary mechanisms.
\end{abstract}

Keywords: Competing Mechanisms, Exclusive Competition, Incomplete Information.

JEL Classification: D82.

${ }^{*}$ We thank Gorkem Celik, Alfredo Di Tillio, Thomas Mariotti, the editor Francoise Forges, an associate editor, and three anonymous referees for extremely valuable comments. We also thank the seminar audiences at Università di Roma Tor Vergata, Università Gabriele D'Annunzio Pescara-Chieti, Università di Sassari, IPAG (Paris), as well as the participants at the 2013 ASSET conference, the 2014 John Cabot Economic Theory workshop and 2015 Society for Economic Design conference for useful discussions. Financial support from MIUR (PRIN 2011 and PRIN 2015) and from the SCOR-TSE Chair is gratefully acknowledged.

${ }^{\dagger}$ Toulouse School of Economics (CNRS), and DEF Università di Roma Tor Vergata; andrea.attar@tse-fr.eu

${ }^{\ddagger}$ DEF Università di Roma Tor Vergata; eloisa.campioni@uniroma2 . it

${ }^{\S}$ IPAG Business School; gwenael.piaser@ipag.fr

CORRESPONDING AUTHOR : Andrea Attar, Toulouse School of Economics, 21 allée de Brienne, 31000 Toulouse (France). 


\section{Introduction}

We study competing-mechanism games of incomplete information in which competition is exclusive: contracts incorporate exclusivity clauses and they are fully enforced. Thus, upon observing her private information and the publicly observable mechanisms, each agent can participate and communicate with at most one principal. Each principal fully observes the set of agents who participate with him, and agents take no hidden actions. Final allocations are determined by the contracts that principals independently sign with agents.

Exclusive competition is at the hearth of most theoretical analyses of markets with privately informed agents. This assumption plays a fundamental role in competing auctions (McAfee, 1993), competitive screening (Rothschild and Stiglitz, 1976), competitive search (Guerrieri et al., 2010), and competing hierarchies (Martimort, 1996) models, among many others. At the same time, letting firms compete over exclusive contracts is regarded as a convenient way to represent full observability of agents' trades in the extension of general equilibrium theory to private information economies (Prescott and Townsend, 1984; Bisin and Gottardi, 2006).

Despite the prominent role of these economic settings, we still lack a general characterization of equilibrium mechanisms under exclusive competition. Indeed, the approaches above share the restriction to standard direct mechanisms: each principal commits to incentive schemes that associate his decisions to the array of exogenous types reported by agents. ${ }^{1}$ In addition, they focus on incentive compatible mechanisms which induce agents to truthfully reveal their types to the principal they participate with. Yet, few, if any, theoretical arguments have been developed to support these choices. If competition is exclusive, to what extent can one safely restrict attention to direct mechanisms and to agents' truthful behaviors? The present paper contributes to answer this question by setting up a general competing-mechanism setting of exclusive competition, and introducing a solution concept that formalizes the above restrictions. We provide two main results.

First, we show that the restriction to direct mechanisms involves a loss of generality. We exhibit a pure strategy equilibrium outcome of an incomplete information game, in which principals post indirect mechanisms that cannot be reproduced in the game in which they post direct ones. The example is casted in a stylized competitive insurance setting in which two insurance companies compete to serve two privately informed consumers. We show that the communication made available by indirect mechanisms allows to support a monopolistic allocation at equilibrium. Indeed, indirect mechanisms generate threats that the monopolistic principal cannot reproduce by relying on simple direct mechanisms.

Second, we formalize the restrictions made in economic applications by introducing the notion

\footnotetext{
${ }^{1}$ In such direct mechanisms, agents only communicate to principals their exogenous private information (type), but not the market information generated by the presence of several competing mechanisms. Modeling this additional communication may require very complex message spaces. Epstein and Peters (1999) are the first to characterize the universal mechanisms which allow to report all relevant information, therefore formalizing an extended notion of type for the agents. This abstract construction, however, has little bite in applications, as extensively discussed by Peters (2014).
} 
of truth-telling strongly robust equilibrium. In a competing-mechanism game in which principals post direct mechanisms, a truth-telling strongly robust equilibrium is a (subgame perfect) equilibrium in which agents are truthful on the equilibrium path, and no principal has a profitable deviation regardless of the continuation equilibrium that agents may play. We investigate whether the corresponding outcomes survive if a principal deviates to general mechanisms. This amounts to analyzing the implications of enlarging the strategy space of a single principal, holding fixed the behavior of his rivals. We prove that any truth-telling strongly robust equilibrium in which principals play pure strategies survives if a principal deviates to any indirect mechanism. As will be discussed in Section 2, this provides a rationale for the restrictions postulated in applications. The result crucially relies on the assumption of exclusive competition, which ties an agent's communication to her participation choice. This is shown by means of a two-principals two-agents example, in which agents' communication is unrestricted: each type of each agent can participate with at most one principal, and can send non-degenerate messages to both of them. In the example, fixing the mechanism of one principal, we can hence construct a deviation to an indirect mechanism of the other principal and an induced equilibrium of the agents' game, generating a profile of decisions which cannot be reproduced by a deviation to a direct mechanism and an associated truthful report for the agents.

Related literature. This paper contributes to the literature on equilibrium characterization in competing-mechanism games. Several game-theoretic examples have shown that direct mechanisms fail to provide a full characterization of equilibrium outcomes. To get the result, however, they crucially exploit either the fact that each agent participates with many principals at a time (Peters, 2001; Martimort and Stole, 2002), or that an agent participating with a given principal can also communicate with the principals who post the competing mechanisms (Epstein and Peters, 1999). We instead consider competing mechanisms under exclusive competition. That is, agents participate with at most one principal and their communication decisions are restricted: each type of each agent sends informative messages only to the principal it participates with. In this context, we show the existence of equilibrium outcomes that cannot be supported by direct mechanisms.

Another key issue for equilibrium characterization is whether equilibria supported by direct mechanisms are robust against principals' deviations towards indirect ones. A positive result in this direction is provided by Peters (2003) under common agency. He shows that, in single-agent models of nonexclusive competition, a principal cannot profitably deviate from a pure strategy, truthtelling, equilibrium by using arbitrary indirect mechanisms. ${ }^{2}$ The result does not typically extend to multiple agents. ${ }^{3}$ An exception is provided by Han (2007) who considers competing-mechanism games of complete information, and shows that each strongly robust equilibrium outcome of the direct mechanism game survives against principals' unilateral deviations to indirect mechanisms.

\footnotetext{
${ }^{2}$ See Theorem 2 in Peters (2003).

${ }^{3}$ Peters (2004) and Attar et al. (2012) provide examples of multiple-agent settings with moral hazard in which agents participate with all principals at a time and equilibria supported by direct mechanisms fail to be robust.
} 
We extend Han (2007) analysis to the incomplete information case by proving a robustness result for exclusive competition settings. Truth-telling strongly robust equilibria survive against principals' deviations to more complex communication schemes. In a similar exclusive competition framework, Peck (1997) reaches a negative conclusion: given the mixed strategy of one principal, his opponent can post a mechanism inducing a continuation equilibrium which cannot be reproduced when agents behave truthfully. Our Proposition 1 hence clarifies that Peck (1997)'s insight fundamentally relies on principals playing mixed strategies at equilibrium.

The paper is organized as follows: Section 2 develops a general model of competing mechanisms under exclusive competition. Section 3 provides an example showing the limited power of direct mechanisms. Section 4 proves equilibrium robustness, and clarifies the role of exclusive competition. Section 5 concludes.

\section{The model}

\subsection{Competing mechanisms under exclusive competition}

We refer to a scenario in which several principals (indexed by $j \in \mathcal{J}=\{1, \ldots, J\}$ ) contract with several agents (indexed by $i \in \mathcal{I}=\{1, \ldots, I\}$ ). Each agent $i$ has private information about her type $\omega^{i} \in \Omega^{i}$. Each $\Omega^{i}$ is taken to be finite, and we let $p(\omega)$ be the probability of the array of types $\omega=\left(\omega^{1}, \ldots, \omega^{I}\right) \in \Omega=\underset{i \in \mathcal{I}}{\times} \Omega^{i}$

We let $x_{j} \in X_{j}$ be a decision available to principal $j$, with $X_{j}$ being a finite set for every $j \in \mathcal{J}$. Similarly, $a_{j}^{i} \in A_{j}^{i}=\{Y, N\}$ represents the decision of agent $i$ to participate with principal $j$, in which $N$ stands for not participating. We take $v_{j}: X \times A \times \Omega \rightarrow \mathbb{R}$ and $u^{i}: X \times A \times \Omega \rightarrow \mathbb{R}$ to be the payoff functions of principal $j$ and of agent $i$, respectively, with $X=\underset{j \in \mathcal{J}}{\times} X_{j}$ and $A=\underset{i \in \mathcal{I}}{\times} \underset{j \in \mathcal{J}}{\times} A_{j}^{i}$.

Communication occurs via the public mechanisms posted by principals, and via the messages sent by agents: each agent $i$ sends a private message $m_{j}^{i} \in M_{j}^{i}$ to principal $j$. We let each $M_{j}^{i}$ be finite, include the element $\{\emptyset\}$ corresponding to the information "agent $i$ does not communicate with principal $j "$, and be sufficiently rich that $\Omega^{i} \subset M_{j}^{i}$ for every $i$ and every $j .{ }^{4}$

Each principal perfectly observes the set of agents who participate with him. Hence, principal $j$ can make his decisions contingent on the array of messages $m_{j}$ he receives, with $m_{j}=$ $\left(m_{j}^{1}, m_{j}^{2}, \ldots, m_{j}^{I}\right) \in M_{j}=\underset{i \in \mathcal{I}}{\times} M_{j}^{i}$, and on the array of participation decisions $a_{j}=\left(a_{j}^{1}, a_{j}^{2}, \ldots, a_{j}^{I}\right) \in$ $A_{j}=\underset{i \in \mathcal{I}}{\times} A_{j}^{i}=\{Y, N\}^{|\mathcal{I}|}$. Formally, we say that a mechanism posted by principal $j$ is a mapping $\gamma_{j}: M_{j} \times A_{j} \rightarrow \Delta\left(X_{j}\right)$. We refer to $\gamma_{j}\left(m_{j}, a_{j}\right)$ as to the probability distribution over $X_{j}$ induced by the array of messages and actions $\left(m_{j}, a_{j}\right)$, and to $\gamma_{j}\left(x_{j} \mid m_{j}, a_{j}\right)$ as to the probability that $\gamma_{j}\left(m_{j}, a_{j}\right)$ assigns to the decision $x_{j} \in X_{j}$. We let $\Gamma_{j}$ be the set of mechanisms available to principal $j$, and denote $\Gamma=\underset{j \in \mathcal{J}}{\times} \Gamma_{j}$. Observe that each $\Gamma_{j}=\left(\Delta\left(X_{j}\right)\right)^{\left|M_{j} \times A_{j}\right|}$ is compact in the product topology.

\footnotetext{
${ }^{4}$ This framework can be extended to situations in which the relevant sets of types, messages, and decisions are infinite. See, for example, the complete information setting of Han (2007).
} 
The competing-mechanism game unfolds as follows. First, principals simultaneously commit to mechanisms, next agents' types are drawn. Given the observed mechanisms $\gamma=\left(\gamma_{1}, \gamma_{2}, \ldots, \gamma_{J}\right)$ and having received their private types $\omega=\left(\omega^{1}, \ldots, \omega^{I}\right)$, agents simultaneously take a participation and a communication decision with every principal. After the agents' choices have been made, principals' decisions are implemented, lotteries realize and payoffs are distributed.

A pure strategy for principal $j$ is hence a mechanism $\gamma_{j} \in \Gamma_{j}$, and a mixed strategy is a probability distribution over $\Gamma_{j}$. A strategy for each agent $i$ associates to every profile of posted mechanisms a joint participation and communication decision, for each value of her type. Exclusive competition implies that each type of each agent can participate with at most one principal and meaningfully communicate only with him. The following assumption captures this restriction.

Assumption E. The set of participation and communication decisions for each agent $i$ is $S^{i}=\left\{\left(m^{i}, a^{i}\right) \in M^{i} \times A^{i}: a_{j}^{i}=Y\right.$ for at most one $j$ and $m_{j}^{i}=\emptyset$ iff $\left.a_{j}^{i}=N\right\}$, with $M^{i}=\underset{j \in \mathcal{J}}{\times} M_{j}^{i}$ and $A^{i}=\underset{j \in \mathcal{J}}{\times} A_{j}^{i}$.

Under Assumption E, a strategy for agent $i$ is a measurable mapping, $\lambda^{i}: \Gamma \times \Omega^{i} \rightarrow \Delta\left(S^{i}\right)$, that associates to each type and to each array of posted mechanisms a probability distribution over such restricted decisions. We denote $\lambda^{i}\left(\gamma, \omega^{i}\right)$ the joint probability distribution over $\left(m^{i}, a^{i}\right) \in S^{i}$ for agent $i$ of type $\omega^{i}$ given $\gamma$, and $\lambda^{i}\left(m^{i}, a^{i} \mid \gamma, \omega^{i}\right)$ the (joint) probability assigned to the pair $\left(m^{i}, a^{i}\right)$ by $\lambda^{i}\left(m^{i}, a^{i}\right)$. The corresponding expected payoff to type $\omega^{i}$ of agent $i$ is:

$$
U^{i}\left(\gamma, \lambda ; \omega^{i}\right)=\sum_{\Omega^{-i}} \sum_{M \times A} \sum_{X} u^{i}\left(x, a, \omega^{i}, \omega^{-i}\right) \prod_{j \in \mathcal{J}} \gamma_{j}\left(x_{j} \mid m_{j}, a_{j}\right) \prod_{h \in \mathcal{I}} \lambda^{h}\left(m^{h}, a^{h} \mid \gamma, \omega^{h}\right) p\left(\omega^{-i} \mid \omega^{i}\right)
$$

where $\lambda=\left(\lambda^{1}, \lambda^{2}, \ldots, \lambda^{I}\right)$, and $p\left(\omega^{-i} \mid \omega^{i}\right)$ is the conditional probability of $\omega^{-i}$ given $\omega^{i}$.

Similarly, the expected payoff to principal $j$ when he plays $\gamma_{j}$ against his opponents' strategies $\gamma_{-j}$ and the agents' strategy profile $\lambda$ is given by:

$$
V_{j}\left(\gamma_{j}, \gamma_{-j}, \lambda\right)=\sum_{\Omega} \sum_{A} \sum_{X} v_{j}(x, a, \omega) \sum_{M} \prod_{k \in \mathcal{J}} \gamma_{k}\left(x_{k} \mid m_{k}, a_{k}\right) \prod_{i \in \mathcal{I}} \lambda^{i}\left(m^{i}, a^{i} \mid \gamma, \omega^{i}\right) p(\omega)
$$

We let $G^{\Gamma}$ be the competing-mechanism game induced by a given $\Gamma{ }^{5}$ As in Epstein and Peters (1999) and Han (2007), we focus on subgame perfect Nash equilibria (SPNE) of the game $G^{\Gamma}$ in which principals play pure strategies. The strategies $(\gamma, \lambda)$ constitute an SPNE of $G^{\Gamma}$ if:

1. $\lambda$ is a continuation equilibrium. That is, for every $\gamma \in \Gamma$, the strategies $\left(\lambda^{i}, \lambda^{-i}\right)$ constitute a Bayes-Nash equilibrium of the agents' game induced by $\gamma$;

\footnotetext{
${ }^{5}$ Observe that in the game $G^{\Gamma}$ mechanisms are defined over $M \times A$, not only on $S$. This is done to consider situations in which Assumption E does not hold, as it is the case for Example 1 in Section 4.
} 
2. given $\gamma_{-j}$ and $\lambda, \gamma_{j} \in \underset{\gamma_{j}^{\prime} \in \Gamma_{j}}{\operatorname{argmax}} V_{j}\left(\gamma_{j}^{\prime}, \gamma_{-j}, \lambda\right)$ for every $j \in \mathcal{J}$.

A mechanism available to principal $j$ is direct if agents can only communicate their types to principal $j$, i.e. if $M_{j}^{i}=\Omega^{i} \cup\{\emptyset\}$ for every $i$, with $\{\emptyset\}$ representing no communication. We denote $\tilde{\gamma}_{j}: \underset{i \in \mathcal{I}}{\times}\left(\Omega^{i} \cup\{\emptyset\}\right) \times\{Y, N\}^{|\mathcal{I}|} \rightarrow \Delta\left(X_{j}\right)$ a direct mechanism for principal $j$, and we let $\Gamma_{j}^{D} \subseteq \Gamma_{j}$ be the corresponding set of direct mechanisms. ${ }^{6}$ Thus, $\tilde{\gamma}_{j}\left(\omega_{j}, a_{j}\right)$ denotes the probability distribution over $X_{j}$ induced by the array of reports $\omega_{j}$ to principal $j$ and by the participation decisions $a_{j}$ he observes.

We let $G^{D}$ be the game in which principals are restricted to direct mechanisms. In $G^{D}$, agents can only communicate their types to principals. For a given array of mechanism $\gamma_{-j}$ posted by his opponents, a direct mechanism $\tilde{\gamma}_{j}$ is incentive compatible from the point of view of principal $j$ if it induces an equilibrium in the agents' game in which each type of each agent who participates with principal $j$ is truthful to him. Thus, $\tilde{\gamma}_{j}$ can be incentive compatible for the array $\gamma_{-j}$, but not for some other array $\gamma_{-j}^{\prime} \neq \gamma_{-j} .^{7}$

\subsection{Applications}

Our general model encompasses standard economic applications of exclusive competition with incomplete information, as illustrated below.

\section{Competing Auctions}

In a seminal paper, McAfee (1993) analyzes sellers who compete over auctions when buyers' valuation constitute their private information. Peters (1997) shows that in a decentralized market with many sellers and buyers, second-price auctions with reserve price equal to marginal cost arise as equilibrium mechanisms. In these settings, sellers simultaneously and anonymously post auctions and each type of each buyer chooses at most one auction to participate in. A seller only receives relevant messages from the buyers who participate in his auction, which guarantees that Assumption E is satisfied. Each seller $j$ is assumed to post direct mechanisms, that associate to the valuations reported by each type $\omega^{i} \in \Omega^{i}=[0,1]$ of each buyer $i \in \mathcal{I}$ who participates in his auction a trading probability and an expected price. A pure strategy for seller $j$ is a mechanism $\tilde{\gamma}_{j}: 2^{|\mathcal{I}|} \times([0,1] \cup \emptyset)^{|\mathcal{I}|} \rightarrow[0,1]^{\left|\mathcal{I}_{j}\right|} \times \mathbb{R}^{\left|\mathcal{I}_{j}\right|}$, with $\mathcal{I}_{j} \subseteq \mathcal{I}$ being the set of buyers participating in auction $j$ and with the assumption that a buyer who does not participate with principal $j$ can only send him the $\emptyset$ message. At equilibrium, buyers behave truthfully: given its participation decision, it is a dominant strategy for each type of each buyer to truthfully report its private valuation in

\footnotetext{
${ }^{6}$ Since $\Omega^{i} \subset M_{j}^{i}$ for each $i$ and $j$, a direct mechanism $\tilde{\gamma}_{j}$ could be specified as a (degenerate) indirect one in which, for each $m_{j} \notin \Omega, \tilde{\gamma}_{j}\left(m_{j}, a\right)=\tilde{\gamma}_{j}(\omega, a)$ for some $\omega \in \Omega$. That is, as in Peters (2001) and Han (2007), allowing for additional messages does not enlarge the set of principal $j$ 's decisions made available by $\tilde{\gamma}_{j}$.

${ }^{7}$ This possibility is already acknowledged in the competing-mechanism setting introduced by Myerson (1982, p.77). See also McAfee (1993, p. 1288).
} 
a second-price auction. Furthermore, when considering seller $j$ 's deviations, attention is restricted to mechanisms inducing truthful reports to the deviator. ${ }^{8}$ Finally, equilibria are constructed in such a way that, if a seller deviates to a new auction mechanism, then buyers coordinate their participation decisions selecting the most favourable continuation equilibrium for the deviator. ${ }^{9}$ The model of this section also adapts to the competing auctions settings of Peters and Severinov (1997), Burguet and Sakovics (1999), Viràg (2010), Han (2015), Peck (2015).

\section{Competitive Insurance}

In their canonical analysis, Rothschild and Stiglitz (1976) study strategic competition between insurance companies for the exclusive right to serve several ex-ante identical agents. Each agent's type space is $\Omega=\left\{\omega^{L}, \omega^{H}\right\}$. Uncertainty is idiosyncratic: each customer faces a binary risk on her endowment $e \in\left\{e_{b}, e_{g}\right\}$. Let $f_{b}(\omega)$ be the probability of the individual state $b$ for an agent of type $\omega \in \Omega$, and $f_{g}(\omega)=1-f_{b}(\omega)$. These random variables are independently distributed across all agents and identically distributed across agents of the same type. Each agent privately observes her type. The payoff to an agent of type $\omega$ is $f_{b}(\omega) u\left(e_{b}+d_{b}\right)+\left(1-f_{b}(\omega)\right) u\left(e_{g}+d_{g}\right)$, where $\left(d_{b}, d_{g}\right) \in \mathbb{R}^{2}$ is the state-contingent coverage purchased from the company it participates with. Attention is restricted to the game in which insurers post (deterministic) direct mechanisms which assign to each agent a pair of state-contingent insurance contracts, as a function of her participation decision and declared type. In addition, such mechanisms induce agents to be truthful to the company they participate with. ${ }^{10}$ Communication is only allowed between each insurer and its customers, implying that Assumption E is satisfied.

\section{Competitive Search}

The model above has been subsequently extended to analyze the interaction between pricing and trading probability. Inderst and Wambach (2002) study a competitive market in which privately informed workers apply to firms who face capacity constraints, and may therefore end up being rationed. The type set of each worker is $\Omega=\left\{\omega^{L}, \omega^{H}\right\}$, and $\omega^{H}$ is the high-ability type. Firms compete by offering price-quantity contracts and workers apply to at most one firm. A single worker can only communicate with a firm when applying for its vacancies. Assumption $\mathrm{E}$ is hence satisfied if we interpret the application decision as participation. Each firm faces an exogenous

\footnotetext{
${ }^{8}$ See, as a reference, McAfee (1993, p. 1288), and Peters (1997, pp. 101-102).

${ }^{9}$ This equilibrium restriction first appears in the competing sellers framework of McAfee (1993, pp. 1288-1290). In the words of Peters and Severinov (1997): "McAfee makes the optimistic assumption that buyers will select the deviator with a probability that maximizes the deviator's profit (subject to the constraint that buyers remain indifferent between the deviator's mechanism and the fixed alternative payoff)." The same restriction is, for instance, incorporated in the notion of competitive distribution of auctions in Peters (1997, pp.109-110), and Han (2015, p. 209).

${ }^{10}$ This setting can alternatively be formulated in a screening context. In the spirit of the taxation principle (Rochet (1985)), insurers are assumed to post menus including at most two non-degenerate insurance contracts. A menu posted by insurer $j$ provides each agent with the same opportunities she can achieve when adopting a truthful behavior with him.
} 
limit $l>0$ to her hiring capacity. Hence, for a given application decision and a given type, a worker's payoff depends on the contract posted by the firm she applies for and on the relevant rationing probability, which is in turn determined by the application decisions of all workers. The competitive search literature endogenizes this rationing as a byproduct of search frictions and bilateral matching (Guerrieri et al., 2010; Eeckhout and Kircher, 2010). Under competitive search, principals simultaneously post direct mechanisms and each agent applies to at most one of them. A direct mechanism is a mapping $\tilde{\gamma}_{j}: 2^{|\mathcal{I}|} \times(\Omega \cup \emptyset)^{|\mathcal{I}|} \rightarrow X_{j}$, with the additional feature that the implemented decision $x_{j} \in X_{j}$ only depends on the number of applications rather than on the identity of the applicants (anonimity). The ratio between the number of principals posting a given mechanism $\gamma$ and the number of agents applying for it determines the rationing probability $\mu(\gamma)$, which every type of every agent takes as given. On the principals' side, attention is restricted to (direct) mechanisms which induce agents to truthfully report their private information on the equilibrium path. Equilibria are characterized by only considering a subset of all possible principals' deviations, that is, those inducing truth-telling to the deviator. ${ }^{11}$ Eventually, for every deviation $\tilde{\gamma}_{j}^{\prime}$ inducing multiple equilibria among agents, the literature follows McAfee (1993) in assuming that the deviator is optimistic, believing that agents will coordinate on the equilibrium which is most favourable to him. ${ }^{12}$

\section{Competing Hierarchies}

The literature on competition between manufacturer-retailer hierarchies developed, among others, by Martimort (1996) and Gal-Or (1997) considers manufacturers who produce differentiated intermediate goods to be sold to retailers for trade in the consumption markets. ${ }^{13}$ Retailers hold private information on market conditions and each of them is exogenously assigned to only one manufacturer. Strategic interactions between retailers arise because the demand price faced by a retailer of hierarchy $j$ depends on the final output of all hierarchies. A manufacturer only communicates with the retailers in his hierarchy, thus Assumption E is satisfied. At equilibrium, each manufacturer $j$ proposes a direct mechanism that assigns a price-quantity pair $\left(p_{j}(\omega), q_{j}(\omega)\right)$ to each type of retailer of his hierarchy who truthfully reports its type $\omega$. When deviating, each manufacturer $j$ anticipates that all retailers in his hierarchy will be truthful to him. ${ }^{14}$ Such framework can hence be seen as a particular case of our general model in which it is a strictly dominant strategy for every type of every agent to participate with only one principal. This specification, however, minimizes

\footnotetext{
${ }^{11}$ See, among others, Guerrieri, Shimer, and Wright (2010, p. 1829).

${ }^{12}$ In these settings, multiple equilibria typically originate from agents' participation choices. In Eeckhout and Kircher (2010, p. 1361), multiplicity is resolved by letting informed buyers coordinate on the queue length that is best for the deviating seller. In the "market for lemons" scenario analyzed by Gottardi and Auster (2016, pp. 10-11), each buyer expects, in case of multiplicity, that sellers select his preferred buyer/seller ratio.

${ }^{13}$ Different strands of the literature investigate the link between pre-commitment effects and renegotiation (Caillaud et al., 1995), the rationale behind alternative forms of vertical restraints (Gal-Or, 1991), the welfare implications of information sharing (Pagnozzi and Piccolo, 2013). See Rey and Vergé (2008) for a survey.

${ }^{14}$ See, for example, Martimort and Piccolo (2010, p. 209).
} 
the relevant strategic effects of competition over mechanisms under exclusivity.

Despite its large domain of applicability, our analysis does not incorporate exclusive dealing models (O'Brien and Shaffer, 1997; Bernheim and Whinston, 1998), which evaluate the role of exclusive contracts in contexts in which agents' participation choices are, in principle, unrestricted and Assumption E is typically not satisfied.

\subsection{A strategic foundation for exclusive competition}

The notion of direct mechanism we refer to reflects the restriction imposed in economic applications: agents only need to report their exogenous type. The above discussion has, in addition, identified three key features of the characterizations provided in such applications.

a) Each principal posts an incentive compatible mechanism at equilibrium.

b) Attention is restricted to such mechanisms when examining unilateral deviations. That is, a deviating principal anticipates that every agent participating with him behaves truthfully.

c) If a deviation induces multiple equilibria over agents' participation decisions, the most favorable one for the deviating principal is selected.

We here formalize these restrictions in the context of a competing mechanism game introducing the notion of truth-telling strongly robust equilibrium for the direct mechanism game $G^{D}$. A truthtelling equilibrium $(\tilde{\gamma}, \tilde{\lambda})$ is an SPNE of $G^{D}$ in which agents are truthful to principals on path. That is, given $\left(\tilde{\gamma}_{j}, \tilde{\gamma}_{-j}\right)$, the agents' equilibrium strategies $\left(\tilde{\lambda}^{i}, \tilde{\lambda}^{-i}\right)$ prescribe that every type of every agent is truthful to the principal it participates with. In other words, from the point of view of each principal $j$, the mechanism $\tilde{\gamma}_{j}$ is incentive compatible given the mechanisms $\tilde{\gamma}_{-j}$ posted by his opponents. This concept imposes no restrictions on agents' behaviors following any principal's deviation. A strongly robust equilibrium $(\tilde{\gamma}, \tilde{\lambda})$ is an $\operatorname{SPNE}$ of $G^{D}$, in which for every $j \in \mathcal{J}$,

$$
V_{j}\left(\tilde{\gamma}_{j}, \tilde{\gamma}_{-j}, \tilde{\lambda}\right) \geq V_{j}\left(\tilde{\gamma}_{j}^{\prime}, \tilde{\gamma}_{-j}, \tilde{\lambda}^{\prime}\right) \quad \forall \tilde{\gamma}_{j}^{\prime} \in \Gamma_{j}^{D}, \forall \tilde{\lambda}^{\prime}
$$

with $\tilde{\lambda}^{\prime}$ being a continuation equilibrium relative to $\left(\tilde{\gamma}_{j}^{\prime}, \tilde{\gamma}_{-j}\right)$. Hence, in a strongly robust equilibrium no principal has an individually profitable deviation regardless of the equilibrium selected by agents in the game induced by the deviation. ${ }^{15}$

The remaining of this paper evaluates the rationale for letting principals post direct mechanisms, and for restricting to truth-telling strongly robust equilibria. Specifically, we show that any such equilibrium is robust against unilateral deviations to indirect mechanisms. In establishing the result we show that players' decisions induced by every such deviation can be reproduced by a deviation

\footnotetext{
${ }^{15}$ In competing-mechanism settings, this notion has first been introduced by Epstein and Peters (1999), and then applied to single-agent contexts by Peters (2001) and to multiple-agent contexts by Han (2007).
} 
to a direct mechanism and an induced equilibrium in which agents are truthful to the deviator. This reconciles our strategic approach with all three features exhibited by economic applications.

\section{Direct mechanisms under exclusive competition}

This section establishes that there exist pure strategy equilibrium outcomes of some game $G^{\Gamma}$ that cannot be supported in the corresponding direct mechanism game $G^{D}$.

Consider a version of the competitive insurance model described in Section 2.2. There are two identical principals (insurers) who compete for the exclusive right to serve two identical agents (costumers). An insurer can provide a state-contingent coverage $\left(d_{b}, d_{g}\right) \in \mathcal{D}$ to each of the customers who participate with him. The set of feasible insurance contracts $\mathcal{D} \subset \mathbb{R}_{+}^{2}$ is compact. The payoff to type $\omega \in\left\{\omega^{L}, \omega^{H}\right\}$ of each agent $i=1,2$ is

$$
U\left(d_{b}, d_{g} ; \omega\right)=f_{b}(\omega) u\left(e_{b}+d_{b}\right)+\left(1-f_{b}(\omega)\right) u\left(e_{g}+d_{g}\right),
$$

with $\left(e_{b}, e_{g}\right)$ such that $e_{b}<e_{g}$ being its state-contingent endowment. ${ }^{16}$ For each $\omega$, the function $U(., . ; \omega)$ is twice continuously differentiable, strictly quasi-concave and the standard single-crossing property is satisfied. We let $\omega^{H}$ be the "high risk" type: one hence has $f_{b}\left(\omega^{H}\right)>f_{b}\left(\omega^{L}\right)$ for each agent.

The corresponding payoff that each principal $j=1,2$ earns on each agent of type $\omega$ he serves is $-\left(f_{b}(\omega) d_{b}+\left(1-f_{b}(\omega)\right) d_{g}\right) .{ }^{17}$ We say that $\left(d_{b}^{*}(\omega), d_{g}^{*}(\omega)\right)$ is a fairly priced full insurance contract for type $\omega \in\left\{\omega^{L}, \omega^{H}\right\}$ if $e_{b}+d_{b}^{*}(\omega)=e_{g}+d_{g}^{*}(\omega)$ and $-f_{b}(\omega) d_{b}^{*}(\omega)-\left(1-f_{b}(\omega)\right) d_{g}^{*}(\omega)=0$. Since $f_{b}\left(\omega^{H}\right)>f_{b}\left(\omega^{L}\right)$, we also have that $-f_{b}\left(\omega^{H}\right) d_{b}^{*}\left(\omega^{L}\right)-\left(1-f_{b}\left(\omega^{H}\right)\right) d_{g}^{*}\left(\omega^{L}\right)<0$ : the contract $\left(d_{b}^{*}\left(\omega^{L}\right), d_{g}^{*}\left(\omega^{L}\right)\right)$ yields losses to principals when it is traded by a high risk type.

In such a scenario, a natural candidate for a pure strategy equilibrium is the Rothschild and Stiglitz (1976) allocation. That is, each high risk type of each agent gets the full insurance contract $\left(d_{b}^{*}\left(\omega^{H}\right), d_{g}^{*}\left(\omega^{H}\right)\right)$ from the company it participates with, its incentive constraint binds, and companies earn zero profit on each traded contract.

We show that, if principals can use arbitrary indirect mechanisms, additional outcomes can be supported at equilibrium. Consider, in particular, the situation in which each type of each agent participates with the same principal, say $P 1$, and focus on the monopolistic allocation characterized by Stiglitz (1977), and Schlee and Chade (2012). In this allocation, each low risk type $\omega^{L}$ is left at its reservation utility. That is, the coverage $\left(d_{b}^{M}\left(\omega^{L}\right), d_{g}^{M}\left(\omega^{L}\right)\right)$ is such that $U\left(d_{b}^{M}\left(\omega^{L}\right), d_{g}^{M}\left(\omega^{L}\right) ; \omega^{L}\right)=U\left(0,0, \omega^{L}\right)=f_{b}\left(\omega^{L}\right) u\left(e_{b}\right)+\left(1-f_{b}\left(\omega^{L}\right)\right) u\left(e_{g}\right)$. The coverage for each high risk type $\left(d_{b}^{M}\left(\omega^{H}\right), d_{g}^{M}\left(\omega^{H}\right)\right)$ is such that her incentive constraint binds and it ends up being

\footnotetext{
${ }^{16}$ Since, for a given type and for a given coverage, agent 1 and 2 have the same (expected) payoff, we do not specify the superscript $i$ in equation (4) to simplify exposition.

${ }^{17}$ When the number of agents gets large, this becomes a standard model of competitive economies under adverse selection (Bisin and Gottardi, 2006).
} 
fully insured. That is, $U\left(d_{b}^{M}\left(\omega^{H}\right), d_{g}^{M}\left(\omega^{H}\right) ; \omega^{H}\right)=U\left(d_{b}^{M}\left(\omega^{L}\right), d_{g}^{M}\left(\omega^{L}\right) ; \omega^{H}\right)$, with $e_{b}+d_{b}\left(\omega^{H}\right)=$ $e_{g}+d_{g}\left(\omega^{H}\right)$. This in turn guarantees that $-\left(f_{b}(\omega) d_{b}^{M}(\omega)+\left(1-f_{b}(\omega)\right) d_{g}^{M}(\omega)\right)>0$ for each $\omega$.

This outcome can be supported in a pure strategy equilibrium of a competing-mechanism game $G^{\Gamma}$ in which each agent's message set contains, beyond her individual types and the degenerate message $\emptyset$, the two additional messages $m$ and $m^{\prime}$. Specifically, let $P 1$ propose the following mechanism:

i) if both agents participate with him sending a pair of messages $(\omega, \tilde{\omega}) \in\left\{\omega^{L}, \omega^{H}\right\}^{2}$, he provides the coverages $\left(d_{b}^{M}(\omega), d_{g}^{M}(\omega)\right)$ and $\left(d_{b}^{M}(\tilde{\omega}), d_{g}^{M}(\tilde{\omega})\right)$, respectively;

ii) if both agents participate with him sending a pair of messages in the set $\left\{m, m^{\prime}\right\}^{2}$, he provides the coverage $\left(\hat{d}_{b}\left(\omega^{L}\right), \hat{d}_{g}\left(\omega^{L}\right)\right)=\underset{d \in D}{\operatorname{argmax}} U\left(d_{b}, d_{g} ; \omega^{L}\right)$ to each agent sending $m$, and the coverage $\left(\hat{d}_{b}\left(\omega^{H}\right), \hat{d}_{g}\left(\omega^{H}\right)\right)=\underset{d \in D}{\operatorname{argmax}} U\left(d_{b}, d_{g} ; \omega^{H}\right)$ to each agent sending $m^{\prime}$;

iii) for every other combination of participation choices and messages, he provides the coverage $\left(d_{b}^{M}(\omega), d_{g}^{M}(\omega)\right)$ to the agent sending the message $\omega$ and the coverage $d_{b}(\emptyset)=d_{g}(\emptyset)=0$ to the agent sending the messages $m, m^{\prime}$ or $\emptyset$.

In addition, let the other principal $P 2$ offer the null coverage $d_{b}(\emptyset)=d_{g}(\emptyset)=0$ for every participation and communication choices.

Given these mechanisms, there is an equilibrium of the agents' game in which each type of each agent participates with $P 1$ and truthfully reveals its private information. ${ }^{18}$ Clearly, $P 1$ has no incentive to deviate since he is earning the monopolistic profit. We therefore only have to consider deviations of $P 2$.

Consider then the continuation game induced by a deviation of $P 2$ to some mechanism $\gamma_{2}^{\prime}$. If type $\omega^{L}\left(\omega^{H}\right)$ of an agent participates with $P 1$ sending him the message $m\left(m^{\prime}\right)$, then any type $\omega \in\left\{\omega^{L}, \omega^{H}\right\}$ of the other agent can get its maximal payoff $U\left(\hat{d}_{b}(\omega), \hat{d}_{g}(\omega) ; \omega\right)$ by sending the message $m$ if $\omega=\omega^{L}$, or $m^{\prime}$ if $\omega=\omega^{H}$, to $P 1$. For each $\gamma_{2}^{\prime}$ there is hence an equilibrium in which each type $\omega$ of each agent participates with $P 1$ and receives the contract $\hat{d}(\omega)$. We then construct the agents' equilibrium strategies so that, following any such $P 2$ 's deviation, each type $\omega^{L}\left(\omega^{H}\right)$ participates with $P 1$ sending the message $m\left(m^{\prime}\right)$. Thus, there is no deviation in which $P 2$ can profitably attract at least one agent.

We now show that the same monopolistic coverage cannot be supported at equilibrium in $G^{D}$, in which principals post direct mechanisms. When posting a (deterministic) direct mechanism, a principal provides a coverage $\left(d_{b}(\omega), d_{g}(\omega)\right)$ to each agent who participates with him and reports $\omega \in\left\{\omega^{L}, \omega^{H}\right\}$, and the null coverage $\left(d_{b}(\emptyset), d_{g}(\emptyset)\right)=(0,0)$ to each agent who does not participate with him. For $P 1$ to achieve the above monopolistic allocation, he must provide the coverages

\footnotetext{
${ }^{18}$ This follows from $i$ ) and from the fact that, by construction, the monopolistic allocation is incentive compatible.
} 
$\left(d_{b}^{M}(\omega), d_{g}^{M}(\omega)\right)$ and $\left(d_{b}^{M}(\tilde{\omega}), d_{g}^{M}(\tilde{\omega})\right)$ when the reported types are $(\omega, \tilde{\omega}) .{ }^{19}$ To show that $P 2$ has a profitable deviation, observe that, by continuity and single crossing of each $U(., . ; \omega)$ function, there exists a pair of insurance contracts $\left(d_{b}^{\prime}\left(\omega^{L}\right), d_{g}^{\prime}\left(\omega^{L}\right)\right)$, and $\left(d_{b}^{\prime}\left(\omega^{H}\right), d_{g}^{\prime}\left(\omega^{H}\right)\right)$ in a neighborhood, respectively, of $\left(d_{b}^{M}\left(\omega^{L}\right), d_{g}^{M}\left(\omega^{L}\right)\right)$ and $\left(d_{b}^{M}\left(\omega^{H}\right), d_{g}^{M}\left(\omega^{H}\right)\right)$ such that:

$$
U\left(d_{b}^{\prime}\left(\omega^{L}\right), d_{g}^{\prime}\left(\omega^{L}\right) ; \omega^{L}\right)>\max \left\{U\left(d_{b}^{M}\left(\omega^{L}\right), d_{g}^{M}\left(\omega^{L}\right) ; \omega^{L}\right), U\left(d_{b}^{\prime}\left(\omega^{H}\right), d_{g}^{\prime}\left(\omega^{H}\right) ; \omega^{L}\right)\right\}
$$

and

$$
U\left(d_{b}^{\prime}\left(\omega^{H}\right), d_{g}^{\prime}\left(\omega^{H}\right) ; \omega^{H}\right)>\max \left\{U\left(d_{b}^{M}\left(\omega^{H}\right), d_{g}^{M}\left(\omega^{H}\right) ; \omega^{H}\right), U\left(d_{b}^{\prime}\left(\omega^{L}\right), d_{g}^{\prime}\left(\omega^{L}\right) ; \omega^{H}\right)\right\}
$$

for each agent. That is, each type $\omega$ strictly prefers to purchase $\left(d_{b}^{\prime}(\omega), d_{g}^{\prime}(\omega)\right)$ rather than $\left(d_{b}^{\prime}\left(\omega^{\prime}\right), d_{g}^{\prime}\left(\omega^{\prime}\right)\right)$ which is designed to attract type $\omega^{\prime}$. Its corresponding payoff is greater than the equilibrium one. In addition, the continuity of insurer's profits guarantees that these contracts can be chosen so that:

$$
-f_{b}(\omega) d_{b}^{\prime}(\omega)+\left(1-f_{b}(\omega) d_{g}^{\prime}(\omega)\right)>0
$$

for every $\omega \in\left\{\omega^{L}, \omega^{H}\right\}$. Suppose now that $P 2$ deviates to the direct mechanism which provides the coverage $\left(d_{b}^{\prime}(\omega), d_{g}^{\prime}(\omega)\right)$ to every agent who participates with him and reports $\omega$. Following $P 2$ 's deviation, there is no equilibrium of the agents' game in which both agents participate with $P 1$. Indeed, if one of them does so, (5) and (6) guarantee that the other one strictly prefers to participate with $P 2$. In any equilibrium, at least one agent will therefore participate with $P 2$ with a strictly positive probability. Then, (5) and (6) guarantee that each type of this agent will be truthful to $P 2$. By (7), the deviation yields $P 2$ a strictly positive profit. The result obtains for every possible specification of $P 1$ 's direct mechanism when only one agent participates with him.

1. The example shows that a monopolistic allocation can arise at equilibrium in a standard insurance setting with exclusive competition. This obtains by letting principals use indirect mechanisms, but the same allocation cannot be supported in an equilibrium of $G^{D}$. The (equilibrium) indirect mechanism of $P 1$ makes available a system of threats that allow agents to punish his opponent when he attempts at profitably deviating. Indeed, following any such deviation, there is an equilibrium of the agents' game that keeps $P 2$ excluded from trade and yields every type $\omega$ the coverage $\left(\hat{d}_{b}(\omega), \hat{d}_{g}(\omega)\right)$. Importantly, the threats are effective despite the exclusive nature of competition, captured by Assumption E. Direct mechanisms turn out not to be flexible enough to reproduce all these threats, which shrinks the set of equilibrium outcomes they can support. In this respect, the example extends to exclusive competition contexts the general intuition provided by Peters (2001)

\footnotetext{
${ }^{19}$ As we clarify in the text, our argument does not require to specify the direct mechanism of $P 1$ for the case in which only one agent participates with him. We therefore omit this specification to simplify exposition.
} 
and Martimort and Stole (2002) in single-agent, nonexclusive frameworks. Yet, our result crucially exploits the presence of several agents. If there was only one agent, the threats generated by the indirect mechanism posted by $P 1$ at equilibrium would not be effective. Indeed, if $P 1$ 's mechanism makes available the coverage $\left(\hat{d}_{b}(\omega), \hat{d}_{g}(\omega)\right)$, then type $\omega$ of the agent would simply send the corresponding message on the equilibrium path. ${ }^{20}$

2. The equilibrium (indirect) mechanism of $P 1$ can be naturally interpreted as a recommendation mechanism in the sense of Yamashita (2010). In a recommendation mechanism, agents report their types to each principal they participate with, and recommend him a specific direct mechanism to post. In this interpretation, the mechanism of $P 1$ induces agents to recommend him, on the equilibrium path, to provide $\left(d_{b}^{M}(\omega), d_{g}^{M}(\omega)\right)$ to each agent declaring to be of type $\omega$. The equilibrium is supported by the following out-of-equilibrium behaviors: each type $\omega$ of each agent participates with $P 1$ and recommends him to post the direct mechanism yielding the payoff $U\left(\hat{d}_{b}(\omega), \hat{d}_{g}(\omega) ; \omega\right)$ to each type $\omega$. These threats sustain $P 2$ 's exclusion from trade at equilibrium. ${ }^{21}$

3. The example does not attempt at providing a characterization of the equilibria of $G^{D}$. Yet, as argued above, the allocation characterized by Rothschild and Stiglitz (1976) can be supported in a pure strategy equilibrium of $G^{D}$. In this case, on the equilibrium path, each high risk type of each agent is indifferent between its equilibrium (full) coverage and that purchased by each low risk type. Were a high risk type switching to this alternative insurance policy, the insurer who trades it with some low risk types would incur losses. Such an (optimal) behavior of high risk type customers is hence not compatible with a pure strategy equilibrium. Hence agents coordinate on the continuation equilibrium which is more favorable to the active principals, in line with the strong robustness requirement introduced in Section 2.3.

4. The example is built in a standard insurance setting in which principals (insurers) are restricted to deterministic mechanisms. This may raise the question of whether indirect mechanisms still allow to support additional equilibria when stochastic mechanisms are allowed. In the example, intuition suggests that the profitability of $P 2$ 's deviation in the game $G^{D}$ does not depend on the randomness associated to the threats of $P 1$. A full-fledged example documenting this result in the presence of stochastic mechanisms is provided in Attar et al. (2015).

Overall, the example shows that restricting principals to direct mechanisms involves a loss of generality even in exclusive competition contexts. The result a fortiori applies to the truth-telling, strongly robust equilibria of $G^{D}$ which have been considered in the literature. A possible rationale for the restriction to such equilibria is provided in the next section.

\footnotetext{
${ }^{20}$ For a general analysis of the role of direct mechanisms in exclusive competition settings with a single agent, see the working paper version (Attar et al., 2015).

${ }^{21}$ Yamashita (2010)'s analys is casted in an abstract multi-principal multi-agent setting in which agents participate and communicate with all principals. In his construction, a mechanism is implemented by a principal if it receives the majority of agents' recommendations (votes). He then shows that, if there are at least three agents, recommendation mechanisms can be used to establish a folk-theorem.
} 


\section{Robustness}

From the viewpoint of applications, an important issue is whether truth-telling strongly robust equilibrium outcomes survive to a principal deviating towards general indirect mechanisms. A positive answer to this question would provide some foundation for the restrictions made in economic models of exclusive competition. We establish such a result in Proposition 1.

Proposition 1 Consider any truth-telling, strongly robust equilibrium of the game $G^{D}$ in which principals play pure strategies. Then, under Assumption E, the corresponding outcome can be supported in an equilibrium of any game $G^{\Gamma}$ in which principals play pure strategies.

Proof Let $\left(\tilde{\gamma}^{*}, \tilde{\lambda}^{*}\right)$ be a truth-telling, strongly robust equilibrium of $G^{D}$ and let $\tilde{V}_{j}^{*}$ and $\tilde{U}^{*} i$ be the equilibrium payoffs for every principal $j \in \mathcal{J}$ and every agent $i \in \mathcal{I}$. We show that the corresponding outcome, that is, the probability distributions over players' decisions given agents' types induced by $\left(\tilde{\gamma}^{*}, \tilde{\lambda}^{*}\right)$ can be supported at equilibrium in any game $G^{\Gamma}$.

Specifically, fix a $G^{\Gamma}$ game and let principals post the (degenerate) indirect mechanisms $\tilde{\gamma}^{*}=$ $\left(\tilde{\gamma}_{j}^{*}, \tilde{\gamma}_{-j}^{*}\right)$ and agents play according to the strategies such that:

a.) $\lambda^{* i}\left(\gamma, \omega^{i}\right)=\tilde{\lambda}^{* i}\left(\gamma, \omega^{i}\right) \quad \forall i, \forall \omega^{i}$ and $\forall \gamma \in \Gamma^{D} \subseteq \Gamma$

b.) $\lambda^{*}(\gamma, \omega)$ is an equilibrium of the agents' game induced by $\gamma=\left(\gamma_{j}, \gamma_{-j}\right) \quad \forall \omega$ and $\forall \gamma \notin \Gamma^{D}$.

Thus, in the "enlarged" game in which all mechanisms are feasible, each type $\omega^{i}$ of each agent $i$ takes the same participation and communication decisions that it was taking at the original truthtelling equilibrium of $G^{D}$, when an array of (direct) mechanisms is posted. When, instead, at least one principal posts an indirect mechanism, we let agents coordinate on an arbitrary equilibrium.

To develop the proof, we focus on the situation in which a principal, say the $j$-th one, unilaterally deviates to some $\gamma_{j}^{\prime} \in \Gamma_{j}$, and consider the agents' game induced by the mechanisms $\left(\gamma_{j}^{\prime}, \tilde{\gamma}_{-j}^{*}\right)$. It is convenient to represent the equilibrium behavior of each type $\omega^{i}$ of each agent $i$ in this game in terms of the probability distributions over communication and participation decisions. That is, we denote:

$$
\lambda^{* i}\left(\gamma_{j}{ }^{\prime}, \tilde{\gamma}_{-j}^{*}, \omega^{i}\right)=\mu^{* i}\left(. \mid a_{j}^{i}, a_{-j}^{i}, \gamma_{j}{ }^{\prime}, \tilde{\gamma}_{-j}^{*}, \omega^{i}\right) \otimes \pi^{* i}\left(. \mid \gamma_{j}{ }^{\prime}, \tilde{\gamma}_{-j}^{*}, \omega^{i}\right),
$$

in which $\mu^{* i}\left(. \mid a_{j}^{i}, a_{-j}^{i}, \gamma_{j}^{\prime}, \tilde{\gamma}_{-j}^{*}, \omega^{i}\right)$ represents the conditional equilibrium probability distribution over the message profiles $\left(m_{j}^{i}, m_{-j}^{i}\right)$ that type $\omega^{i}$ of agent $i$ uses if it chooses the participation decisions $\left(a_{j}^{i}, a_{-j}^{i}\right)$ with a strictly positive probability, and $\pi^{* i}\left(. \mid \gamma_{j}^{\prime}, \tilde{\gamma}_{-j}^{*}, \omega^{i}\right)$ represents its (marginal) equilibrium probability distribution over participation decisions $a^{i}=\left(a_{j}^{i}, a_{-j}^{i}\right)$. The following lemma makes use of this representation to establish a characterization result that is key to our argument. 
Lemma 1 Fix a principal $j$ and let principals $-j$ post the direct mechanisms $\tilde{\gamma}_{-j} \in \Gamma_{-j}^{D}$. For each mechanism $\gamma_{j}^{\prime} \in \Gamma_{j}$ posted by principal $j$, let $(\bar{\mu}, \bar{\pi})$ be an equilibrium of the agents' game induced by $\left(\gamma_{j}^{\prime}, \tilde{\gamma}_{-j}\right)$. Then, under Assumption E, there exists a $\tilde{\gamma}_{j}^{\prime} \in \Gamma_{j}^{D}$ and an equilibrium $(\tilde{\mu}, \bar{\pi})$ of the agents' game induced by $\left(\tilde{\gamma}_{j}^{\prime}, \tilde{\gamma}_{-j}\right)$, which reproduce the same probability distribution over principals' decisions as the one implied by $\left(\gamma_{j}^{\prime}, \tilde{\gamma}_{-j}\right)$ and $(\bar{\mu}, \bar{\pi})$. In particular, $\tilde{\mu}$ prescribes to each type $\omega^{i}$ of each agent $i$ to be truthful to principal $j$ when it participates with him.

Proof Suppose that, given the mechanisms $\tilde{\gamma}_{-j} \in \Gamma_{-j}^{D}$, principal $j$ posts the mechanism $\gamma_{j}^{\prime} \in \Gamma_{j}$. In the induced game between agents, let $\bar{\pi}=\left(\bar{\pi}^{1}, \ldots, \bar{\pi}^{I}\right)$ be an (equilibrium) distribution over participation, with $\bar{\pi}^{i}=\bar{\pi}^{i}\left(\cdot \mid \gamma_{j}^{\prime}, \tilde{\gamma}_{-j}, \omega^{i}\right)$, and $\bar{\mu}=\left(\bar{\mu}^{1}, \ldots, \bar{\mu}^{I}\right)$ be an (equilibrium) conditional distribution over messages, with $\bar{\mu}^{i}=\bar{\mu}^{i}\left(. \mid a^{i}, \gamma_{j}^{\prime}, \tilde{\gamma}_{-j}, \omega^{i}\right)$, for every $a^{i}$ in the support of $\bar{\pi}^{i}$, and for every $i \in \mathcal{I}$.

Given the mechanisms $\left(\gamma_{j}^{\prime}, \tilde{\gamma}_{-j}\right)$, for each $\omega \in \Omega$ and for each $a_{j}$ which is played with positive probability in $\bar{\pi}$, let $I_{\omega}\left(a_{j} ; \gamma_{j}^{\prime}, \tilde{\gamma}_{-j}, \bar{\pi}\right)$ be the subset of types that participate with principal $j$, and $\bar{I}_{\omega}\left(a_{j} ; \gamma_{j}^{\prime}, \tilde{\gamma}_{-j}, \bar{\pi}\right)$ be the subset of those who do not participate with principal $j$ when $a_{j}$ is chosen. Hence, the sets $I_{\omega}\left(a_{j} ; \gamma_{j}^{\prime}, \tilde{\gamma}_{-j}, \bar{\pi}\right)$ and $\bar{I}_{\omega}\left(a_{j} ; \gamma_{j}^{\prime}, \tilde{\gamma}_{-j}, \bar{\pi}\right)$ partition the profile of types $\omega=\left(\omega^{1}, \ldots, \omega^{I}\right)$ in terms of their equilibrium participation decisions $\bar{\pi}$. Let $h$ be a generic element of $I_{\omega}\left(a_{j} ; \gamma_{j}^{\prime}, \tilde{\gamma}_{-j}, \bar{\pi}\right)$, indicating that type $\omega^{h}$ of agent $h$ participates with principal $j$. Analogously, let $k$ be a generic element of $\bar{I}_{\omega}\left(a_{j} ; \gamma_{j}^{\prime}, \tilde{\gamma}_{-j}, \bar{\pi}\right)$.

We construct the direct mechanism $\tilde{\gamma}_{j}^{\prime} \in \Gamma_{j}^{D}$ such that:

$$
\tilde{\gamma}_{j}^{\prime}\left(\omega_{j}, a_{j}\right)=\sum_{M_{j}} \sum_{M_{-j}}\left(\prod_{i \in \mathcal{I}} \bar{\mu}^{i}\left(m_{j}^{i}, m_{-j}^{i} \mid a^{i}, \gamma_{j}{ }^{\prime}, \tilde{\gamma}_{-j}, \omega^{i}\right)\right) \gamma_{j}^{\prime}\left(m_{j}, a_{j}\right),
$$

for each $\omega_{j} \in \underset{i \in \mathcal{I}}{\times}\left(\Omega^{i} \cup\{\emptyset\}\right)$, and for every $a_{j} \in A_{j}$ played with positive probability in $\bar{\pi}$. For every $a^{i}$ not in the support of $\bar{\pi}^{i}$, we let $\bar{\mu}^{i}\left(. \mid a^{i}, \gamma_{j}^{\prime}, \tilde{\gamma}_{-j}, \omega^{i}\right)$ be any arbitrary distribution over $M_{j}^{i}$. Recall that since $\tilde{\gamma}_{-j} \in \Gamma_{-j}^{D}$, we have that $M_{j^{\prime}}^{i}=\Omega^{i} \cup\{\emptyset\}$ for every $j^{\prime} \neq j$ and for every $i$.

We now consider the game played by types $\left(\omega^{1}, \ldots, \omega^{I}\right)$ given the mechanisms $\left(\tilde{\gamma}_{j}^{\prime}, \tilde{\gamma}_{-j}\right)$. The remaining of the proof is organized in two steps.

Step 1. Let each type participate according to $\left(\bar{\pi}^{1}, \ldots, \bar{\pi}^{I}\right)$, and communicate according to $\tilde{\mu}^{h}\left(\omega^{h} \mid \tilde{\gamma}_{j}^{\prime}, \tilde{\gamma}_{-j}, \omega^{h}\right)=1$ for every $h \in I_{\omega}\left(a_{j} ; \tilde{\gamma}_{j}^{\prime}, \tilde{\gamma}_{-j}, \bar{\pi}\right)$, and according to $\tilde{\mu}^{k}\left(. \mid \tilde{\gamma}_{j}^{\prime}, \tilde{\gamma}_{-j}, \omega^{k}\right)=$ $\bar{\mu}^{k}\left(. \mid \gamma_{j}^{\prime}, \tilde{\gamma}_{-j}, \omega^{k}\right)$ for every $k \in \bar{I}_{\omega}\left(a_{j} ; \tilde{\gamma}_{j}^{\prime}, \tilde{\gamma}_{-j}, \bar{\pi}\right)$. We show that these behaviors reproduce the probability distribution over principals' decisions induced by $\gamma_{j}^{\prime}$ and $(\bar{\mu}, \bar{\pi})$.

By assumption, $I_{\omega}\left(a_{j} ; \gamma_{j}^{\prime}, \tilde{\gamma}_{-j}, \bar{\pi}\right)=I_{\omega}\left(a_{j} ; \tilde{\gamma}_{j}^{\prime}, \tilde{\gamma}_{-j}, \bar{\pi}\right)=I_{\omega}$ for every $\omega$, and $\bar{I}_{\omega}\left(a_{j} ; \gamma_{j}^{\prime}, \tilde{\gamma}_{-j}, \bar{\pi}\right)=$ $\bar{I}_{\omega}\left(a_{j} ; \tilde{\gamma}_{j}^{\prime}, \tilde{\gamma}_{-j}, \bar{\pi}\right)=\bar{I}_{\omega}$. Assumption E implies that, for each $a_{j}$ played with positive probability in $\bar{\pi}$, the decisions selected by $\tilde{\gamma}_{j}^{\prime}$ for different reported types $\omega_{j}$ only depend on the messages that principal $j$ receives from types in $I_{\omega}$, since all types in $\bar{I}_{\omega}$ send him the (constant) empty message. 
Thus, for every $\omega_{j}$ and given $\tilde{\gamma}_{-j}, \tilde{\gamma}_{j}^{\prime}$ reproduces the probability distribution over principal $j$ 's decisions induced by $\gamma_{j}^{\prime}$ and $(\bar{\mu}, \bar{\pi})$ if each type in $I_{\omega}$ is truthful to him. We can therefore write the right-hand side of (9) as:

$$
\begin{aligned}
\sum_{M_{j}} \sum_{M_{-j}} \gamma_{j}^{\prime}\left(m_{j}, a_{j}\right) \prod_{h \in I_{\omega}} \bar{\mu}^{h}\left(m_{j}^{h}, \emptyset \mid a^{h}, \gamma_{j}{ }^{\prime}, \tilde{\gamma}_{-j}, \omega^{h}\right) \prod_{k \in \bar{I}_{\omega}} \bar{\mu}^{k}\left(\emptyset, m_{-j}^{k} \mid a^{k}, \gamma_{j}{ }^{\prime}, \tilde{\gamma}_{-j}, \omega^{k}\right)= \\
=\sum_{M_{j}} \gamma_{j}^{\prime}\left(m_{j}, a_{j}\right) \prod_{h \in I_{\omega}} \bar{\mu}^{h}\left(m_{j}^{h}, \emptyset \mid a^{h}, \gamma_{j}{ }^{\prime}, \tilde{\gamma}_{-j}, \omega^{h}\right),
\end{aligned}
$$

since $\sum_{M_{-j}} \prod_{k \in \bar{I}_{\omega}} \bar{\mu}^{k}\left(\emptyset, m_{-j}^{k} \mid a^{k}, \gamma_{j}{ }^{\prime}, \tilde{\gamma}_{-j}, \omega^{k}\right)=1$. Since each $k \in \bar{I}_{\omega}$ keeps sending messages to principals $-j$ according to $\bar{\mu}^{k}$, the mechanisms $\left(\tilde{\gamma}_{j}^{\prime}, \tilde{\gamma}_{-j}\right)$, and the agents' behaviors $(\tilde{\mu}, \bar{\pi})$ implement the same probability distribution over all principals' decisions as that implied by $\left(\gamma_{j}^{\prime}, \tilde{\gamma}_{-j}\right)$ and $(\bar{\mu}, \bar{\pi})$.

Step 2. We show that participating according to $\left(\bar{\pi}^{1}, \ldots, \bar{\pi}^{I}\right)$ and communicating according to $\tilde{\mu}^{h}\left(\omega^{h} \mid \tilde{\gamma}_{j}^{\prime}, \tilde{\gamma}_{-j}, \omega^{h}\right)=1$ for every $h \in I_{\omega}$, and according to $\tilde{\mu}^{k}\left(. \mid \tilde{\gamma}_{j}^{\prime}, \tilde{\gamma}_{-j}, \omega^{k}\right)=\bar{\mu}^{k}\left(. \mid \gamma_{j}^{\prime}, \tilde{\gamma}_{-j}, \omega^{k}\right)$ for every $k \in \bar{I}_{\omega}$, constitute an equilibrium.

We first reformulate the implication of the previous step in terms of payoffs. Recall first that the expected equilibrium payoff of type $\omega^{i}$ of agent $i$ in the game induced by $\left(\gamma_{j}^{\prime}, \tilde{\gamma}_{-j}\right)$ is

$U^{i}\left(\gamma_{j}^{\prime}, \tilde{\gamma}_{-j},(\bar{\mu}, \bar{\pi}) ; \omega^{i}\right)=\sum_{\Omega^{-i}} \sum_{A} \sum_{X_{j}} \sum_{X_{-j}} u^{i}\left(x_{j}, x_{-j}, a, \omega\right) \beta^{\bar{\mu}}\left(x_{j}, x_{-j} \mid a, \omega\right) \bar{\pi}\left(a \mid \gamma_{j}{ }^{\prime}, \tilde{\gamma}_{-j}, \omega\right) p\left(\omega^{-i} \mid \omega^{i}\right)$

in which we denoted

$$
\begin{aligned}
& \beta^{\bar{\mu}}\left(x_{j}, x_{-j} \mid a, \omega\right) \equiv\left[\sum_{M_{j}} \sum_{M_{-j}} \tilde{\gamma}_{-j}\left(x_{-j} \mid \omega_{-j}, a_{-j}\right) \gamma_{j}^{\prime}\left(x_{j} \mid m_{j}, a_{j}\right) \prod_{t \in \mathcal{I}} \bar{\mu}^{t}\left(m^{t} \mid a^{t}, \gamma_{j}{ }^{\prime}, \tilde{\gamma}_{-j}, \omega^{t}\right)\right]= \\
= & \sum_{M_{j}} \gamma_{j}^{\prime}\left(x_{j} \mid m_{j}, a_{j}\right) \prod_{h \in I_{\omega}} \bar{\mu}^{h}\left(m_{j}^{h}, \emptyset \mid a^{h}, \gamma_{j}^{\prime}, \tilde{\gamma}_{-j}, \omega^{h}\right) \sum_{M_{-j}} \tilde{\gamma}_{-j}\left(x_{-j} \mid \omega_{-j}, a_{-j}\right) \prod_{k \in \bar{I}_{\omega}} \bar{\mu}^{k}\left(\emptyset, m_{-j}^{k} \mid a^{k}, \gamma_{j}{ }^{\prime}, \tilde{\gamma}_{-j}, \omega^{k}\right)= \\
= & \tilde{\gamma}_{j}^{\prime}\left(x_{j} \mid \omega_{j}, a_{j}\right) \sum_{M_{-j}} \tilde{\gamma}_{-j}\left(x_{-j} \mid \omega_{-j}, a_{-j}\right) \prod_{k \in \bar{I}_{\omega}} \bar{\mu}^{k}\left(\emptyset, m_{-j}^{k} \mid a^{k}, \gamma_{j}{ }^{\prime}, \tilde{\gamma}_{-j}, \omega^{k}\right) .
\end{aligned}
$$

Observe that the first equality in the expression for $\beta^{\bar{\mu}}\left(x_{j}, x_{-j} \mid a, \omega\right)$ obtains by (10) and Assumption E that implies

$$
\sum_{M_{j}} \sum_{M_{-j}} \tilde{\gamma}_{-j}\left(x_{-j} \mid \omega_{-j}, a_{-j}\right) \prod_{t \in \mathcal{I}} \bar{\mu}^{t}\left(m^{t} \mid a^{t}, \gamma_{j}{ }^{\prime}, \tilde{\gamma}_{-j}, \omega^{t}\right)=\sum_{M_{-j}} \tilde{\gamma}_{-j}\left(x_{-j} \mid \omega_{-j}, a_{-j}\right) \prod_{k \in \bar{I}_{\omega}} \bar{\mu}^{k}\left(\emptyset, m_{-j}^{k} \mid a^{k}, \gamma_{j}{ }^{\prime}, \tilde{\gamma}_{-j}, \omega^{k}\right)
$$

for each $-j$ principal. While the second equality obtains by using (9) together with Assumption E. Hence, the payoff to type $\omega^{i}$ of agent $i$ when principal $j$ posts $\tilde{\gamma}_{j}^{\prime}$ and agents play according to 
$(\tilde{\mu}, \bar{\pi})$ is equal to her equilibrium payoff in the game induced by $\left(\gamma_{j}^{\prime}, \tilde{\gamma}_{-j}\right)$. Formally,

$$
U^{i}\left(\gamma_{j}^{\prime}, \tilde{\gamma}_{-j},(\bar{\mu}, \bar{\pi}) ; \omega^{i}\right)=\sum_{\Omega^{-i}} \sum_{A} \sum_{X_{j}} \tilde{u}^{i}\left(x_{j}, a, \omega, \omega_{-j}\right) \tilde{\gamma}_{j}^{\prime}\left(x_{j} \mid \omega_{j}, a_{j}\right) \bar{\pi}\left(a \mid \gamma_{j}{ }^{\prime}, \tilde{\gamma}_{-j}, \omega\right) p\left(\omega^{-i} \mid \omega^{i}\right)
$$

with

$$
\tilde{u}^{i}\left(x_{j}, a, \omega, \omega_{-j}\right) \equiv \sum_{X_{-j}} u^{i}\left(x_{j}, x_{-j}, a, \omega\right) \sum_{M_{-j}} \tilde{\gamma}_{-j}\left(x_{-j} \mid \omega_{-j}, a_{-j}\right) \prod_{k \in \bar{I}_{\omega}} \bar{\mu}^{k}\left(\emptyset, m_{-j}^{k} \mid a^{k}, \gamma_{j}{ }^{\prime}, \tilde{\gamma}_{-j}, \omega^{k}\right)
$$

representing the (indirect) payoff to type $\omega^{i}$ which incorporates the decisions of principals other than $j$ induced by the reports $\omega_{-j}$, given the participation decisions $a_{-j}$.

We now develop the proof by contradiction. Suppose first that type $\omega^{i}$ deviates to $\left(b^{i}, \hat{\omega}^{i}\right)$, with $b^{i} \in \operatorname{supp}\left(\bar{\pi}^{i}\right)$ and $\hat{\omega}^{i} \in \Omega^{i} \cup\{\emptyset\}$. Any such deviation is profitable if

$$
\begin{array}{r}
\sum_{\Omega^{-i}} \sum_{A^{-i}} \sum_{X_{j}} \tilde{u}^{i}\left(x_{j}, b^{i}, a^{-i}, \omega, \hat{\omega}_{-j}\right) \tilde{\gamma}_{j}^{\prime}\left(x_{j} \mid \hat{\omega}_{j}^{i}, \omega_{j}^{-i}, b_{j}^{i}, a_{j}^{-i}\right) \bar{\pi}^{-i}\left(a^{-i} \mid \gamma_{j}^{\prime}, \tilde{\gamma}_{-j}, \omega^{-i}\right) p\left(\omega^{-i} \mid \omega^{i}\right)> \\
>\sum_{\Omega^{-i}} \sum_{A^{-i}} \sum_{X_{j}} \tilde{u}^{i}\left(x_{j}, b^{i}, a^{-i}, \omega, \omega_{-j}\right) \tilde{\gamma}_{j}^{\prime}\left(x_{j} \mid \omega_{j}, b_{j}^{i}, a_{j}^{-i}\right) \bar{\pi}^{-i}\left(a^{-i} \mid \gamma_{j}^{\prime}, \tilde{\gamma}_{-j}, \omega^{-i}\right) p\left(\omega^{-i} \mid \omega^{i}\right)= \\
=U^{i}\left(\gamma_{j}^{\prime}, \tilde{\gamma}_{-j},(\bar{\mu}, \bar{\pi}) ; \omega^{i}\right),
\end{array}
$$

with $b_{j}^{i}$ being the $j$-th element of $b^{i}, \hat{\omega}_{-j}=\left(\hat{\omega}_{-j}^{i}, \omega_{-j}^{-i}\right)$ and

$\tilde{u}^{i}\left(x_{j}, b^{i}, a^{-i}, \omega, \hat{\omega}_{-j}\right) \equiv \sum_{X_{-j}} u^{i}\left(x, b^{i}, a^{-i}, \omega\right) \sum_{M_{-j}} \tilde{\gamma}_{-j}\left(x_{-j} \mid \hat{\omega}_{-j}, b_{-j}^{i}, a_{-j}^{-i}\right) \prod_{k \in \bar{I}_{\omega}, k \neq i} \bar{\mu}^{k}\left(\emptyset, m_{-j}^{k} \mid a^{k}, \gamma_{j}^{\prime}, \tilde{\gamma}_{-j}, \omega^{k}\right)$.

The equality on the right-hand side of (12) holds since $b^{i} \in \operatorname{supp}\left(\bar{\pi}^{i}\right)$. At the deviation stage, every type $\omega^{l} \neq \omega^{i}$ keeps participating according to $\bar{\pi}^{l}$ and communicating according to $\tilde{\mu}^{l}$. Specifically, given Assumption E, it sends the empty message to principals other than $j$ when participating with principal $j$, and the empty message to principal $j$ otherwise. Two cases must be then distinguished. 1.) $b^{i}$ is such that type $\omega^{i}$ participates with principal $j$. Thus, given (9) and (10), (12) can be rewritten as

$$
\begin{gathered}
\sum_{\Omega^{-i}} \sum_{A^{-i}} \sum_{X_{j}} \tilde{u}^{i}\left(x_{j}, b^{i}, a^{-i}, \omega, \omega_{-j}\right)\left[\sum_{M_{j}} \gamma_{j}^{\prime}\left(x_{j} \mid \hat{\omega}_{j}^{i}, m_{j}^{-i}, b_{j}^{i}=Y, a_{j}^{-i}\right) \prod_{h \in I_{\omega}, h \neq i} \bar{\mu}^{h}\left(m_{j}^{h}, \emptyset \mid a^{h}, \gamma_{j}^{\prime}, \tilde{\gamma}_{-j}, \omega^{h}\right)\right] \times \\
\times \bar{\pi}^{-i}\left(a^{-i} \mid \gamma_{j}{ }^{\prime}, \tilde{\gamma}_{-j}, \omega^{-i}\right) p\left(\omega^{-i} \mid \omega^{i}\right)>U^{i}\left(\gamma_{j}^{\prime}, \tilde{\gamma}_{-j},(\bar{\mu}, \bar{\pi}) ; \omega^{i}\right), \quad(13)
\end{gathered}
$$

contradicting that $(\bar{\mu}, \bar{\pi})$ is an equilibrium in the game induced by $\left(\gamma_{j}^{\prime}, \tilde{\gamma}_{-j}\right)$. 
2.) $b^{i}$ is such that type $\omega^{i}$ does not participate with principal $j$. In this case, (12) becomes

$$
\begin{gathered}
\sum_{\Omega^{-i}} \sum_{A^{-i}} \sum_{X_{j}} \tilde{u}^{i}\left(x_{j}, b^{i}, a^{-i}, \omega, \hat{\omega}_{-j}\right)\left[\sum_{M_{j}} \gamma_{j}^{\prime}\left(x_{j} \mid \emptyset, m_{j}^{-i}, b_{j}^{i}=N, a_{j}^{-i}\right) \prod_{h \in I_{\omega}, h \neq i} \bar{\mu}^{h}\left(m_{j}^{h}, \emptyset \mid a^{h}, \gamma_{j}^{\prime}, \tilde{\gamma}_{-j}, \omega^{h}\right)\right] \times \\
\times \bar{\pi}^{-i}\left(a^{-i} \mid \gamma_{j}^{\prime}, \tilde{\gamma}_{-j}, \omega^{-i}\right) p\left(\omega^{-i} \mid \omega^{i}\right)>U^{i}\left(\gamma_{j}^{\prime}, \tilde{\gamma}_{-j},(\bar{\mu}, \bar{\pi}) ; \omega^{i}\right), \quad(14)
\end{gathered}
$$

since type $\omega^{i}$ reports $\emptyset$ to principal $j$, and $\hat{\omega}^{i}$ to one of his opponents. Again, the same contradiction obtains.

Alternatively, suppose that type $\omega^{i}$ deviates to $\left(b^{i}, \hat{\omega}^{i}\right)$, with $b^{i} \notin \operatorname{supp}\left(\bar{\pi}^{i}\right)$ and $\hat{\omega}^{i} \in \Omega^{i} \cup\{\emptyset\}$. Any such deviation is profitable if

$$
\begin{array}{r}
\sum_{\Omega^{-i}} \sum_{A^{-i}} \sum_{X_{j}} \tilde{u}^{i}\left(x_{j}, b^{i}, a^{-i}, \omega, \hat{\omega}_{-j}\right) \tilde{\gamma}_{j}^{\prime}\left(x_{j} \mid \hat{\omega}_{j}^{i}, \omega_{j}^{-i}, b_{j}^{i}, a_{j}^{-i}\right) \bar{\pi}^{-i}\left(a^{-i} \mid \gamma_{j}^{\prime}, \tilde{\gamma}_{-j}, \omega^{-i}\right) p\left(\omega^{-i} \mid \omega^{i}\right)> \\
>\sum_{\Omega^{-i}} \sum_{A^{-i}} \sum_{X_{j}} \tilde{u}^{i}\left(x_{j}, a^{i}, a^{-i}, \omega, \omega_{-j}\right) \tilde{\gamma}_{j}^{\prime}\left(x_{j} \mid \omega_{j}, a_{j}^{i}, a_{j}^{-i}\right) \bar{\pi}^{-i}\left(a^{-i} \mid \gamma_{j}^{\prime}, \tilde{\gamma}_{-j}, \omega^{-i}\right) p\left(\omega^{-i} \mid \omega^{i}\right)= \\
=U^{i}\left(\gamma_{j}^{\prime}, \tilde{\gamma}_{-j},(\bar{\mu}, \bar{\pi}) ; \omega^{i}\right),
\end{array}
$$

with $a_{j}^{i}$ being the $j$-th element of any $a^{i} \in \operatorname{supp}\left(\bar{\pi}^{i}\right)$. Whether $\omega^{i}$ s deviation leads to participating with principal $j$ or not, a contradiction can be established following the same logic illustrated above. Indeed, the direct mechanism $\tilde{\gamma}_{j}^{\prime}$ in (9) is constructed so that, if type $\omega^{i}$ of agent $i$ deviates to some participation decision not in the support of $\bar{\pi}^{i}$, it implements a distribution over principal $j$ 's decisions that $\omega^{i}$ could have achieved in the game induced by $\left(\gamma_{j}^{\prime}, \tilde{\gamma}_{-j}\right)$ by means of some message in $M_{j}^{i}$. This concludes the proof of Lemma 1.

To complete the proof, we employ Lemma 1, fixing the profile $\tilde{\gamma}_{-j}$ to be the equilibrium mechanisms $\tilde{\gamma}_{-j}^{*}$. It remains to show that the mechanisms $\left(\tilde{\gamma}_{j}^{*}, \tilde{\gamma}_{-j}^{*}\right)$ and the strategy $\lambda^{*}$ as constructed in a.) -b.) constitute an equilibrium in $G^{\Gamma}$. Assume, by contradiction, that principal $j$ has a profitable deviation $\gamma_{j}^{\prime} \in \Gamma_{j}$. Then, it must be that

$$
V_{j}\left(\gamma_{j}^{\prime}, \tilde{\gamma}_{-j}^{*}, \lambda^{*}\right)>\tilde{V}_{j}^{*}
$$

which implies that $\gamma_{j}^{\prime} \notin \Gamma_{j}^{D}$ given a.). Then, applying Lemma 1, one can write

$$
V_{j}\left(\gamma_{j}^{\prime}, \tilde{\gamma}_{-j}^{*}, \lambda^{*}\right)=V_{j}\left(\tilde{\gamma}_{j}^{\prime}, \tilde{\gamma}_{-j}^{*}, \tilde{\lambda}\right)>\tilde{V}_{j}^{*}
$$

which contradicts that $\left(\tilde{\gamma}^{*}, \tilde{\lambda}^{*}\right)$ is a strongly robust equilibrium in $G^{D}$.

Proposition 1 establishes a robustness result for truth-telling, strongly robust equilibria: at any such equilibrium, no principal can gain by using more sophisticated mechanisms. It extends to incomplete information games of exclusive competition the robustness result of Han (2007). 
The intuition for the proof can be provided as follows. We first show that for every deviation $\gamma_{j}^{\prime} \in \Gamma_{j}$ and every induced equilibrium of the agents' game, there exists a direct mechanism $\tilde{\gamma}_{j}^{\prime}$ for principal $j$ and an equilibrium of the agents' game induced by $\left(\tilde{\gamma}_{j}^{\prime}, \tilde{\gamma}_{-j}\right)$ that reproduce all decisions implemented by the mechanisms $\left(\gamma_{j}^{\prime}, \tilde{\gamma}_{-j}\right)$ in the original equilibrium. The result, established in Lemma 1, extends the reasoning of Myerson (1982) to a scenario in which agents' participation decisions are strategic. This, in particular, ensures that for each direct mechanism $\tilde{\gamma}_{j}^{\prime}$ and for each principal $j$, it is an equilibrium in the game induced by $\left(\tilde{\gamma}_{j}^{\prime}, \tilde{\gamma}_{-j}\right)$ for each type of each agent to be truthful to principal $j$ when participating with him. Strong robustness of the original equilibrium guarantees that, if principal $j$ could profitably deviate to some $\gamma_{j}^{\prime} \in \Gamma_{j}$, then the corresponding $\tilde{\gamma}_{j}^{\prime}$ would also be a profitable deviation from such an equilibrium of $G^{D}$, which generates a contradiction.

Our proof strategy shows that there is no loss of generality in focusing on principals' deviations to direct mechanisms inducing truthful reports to each deviator. This rationalizes the second feature of the approach followed in applications, as described in Section 2.3. Concerning the third one, it is implied by the strong robustness requirement, which implies that each deviating principal is able to coordinate agents on his most preferred continuation equilibrium.

Three assumptions are key for the proof. The first is that each principal plays a pure strategy at equilibrium. Were principals using mixed strategies, agents would hold some relevant information when taking their participation and communication decisions, based on the observation of the realized lottery posted by the principals. Hence, following Peck (1997), indirect communication would be useful for principals to profitably extract this information.

The second key assumption is the strong robustness of the original equilibrium. If this requirement is not met, agents may be able to coordinate on different continuation equilibria when playing the game $G^{D}$ or any $G^{\Gamma}$ following the same unilateral deviation of a principal. This could allow to construct profitable deviations in $G^{\Gamma} .^{22}$

The last one is Assumption E. As long as each type of each agent sends non trivial messages only to the principal it participates with, and given the mechanisms of his competitors, the decisions implemented by any deviation of principal $j$ only depend on the messages he receives from those types who participate with him. This insight does not extend to the case in which agents' communication is unrestricted. To further clarify the role of Assumption $\mathrm{E}$ in the proof, we reconsider the model of Section 2.1, and remove any restriction on agents' communication. In this situation, from the viewpoint of a given principal, the (non-degenerate) messages that agents send to his competitors can be interpreted as hidden actions. Even though these actions are not directly pay-off relevant, they affect the decisions of his opponents which may impact principal $j$ 's payoff in a way that he cannot control. This principal is effectively facing a form of moral hazard on the agents' side. We

\footnotetext{
${ }^{22}$ When agents' participation is strategic, the strong robustness requirement turns out to be key to establish Proposition 1 even with only one principal. This is shown by means of an example in the working paper version of this work (Attar et al., 2015).
} 
show by means of an example that Lemma 1 cannot be established in the presence of this additional strategic effect. The example is built in an incomplete information context, in which each type of each agent can communicate with every principal independently of its participation choice.

Example 1 Let $I=J=2$. Denote the principals as $P 1$ and $P 2$, and the agents as $A 1$ and A2. The decision sets are $X_{1}=\left\{x_{11}, x_{12}, x_{13}, x_{14}\right\}$ for $P 1$, and $X_{2}=\left\{x_{21}, x_{22}, x_{23}, x_{24}\right\}$ for P2. Each agent can be of two types, with $\Omega^{1}=\Omega^{2}=\left\{\omega^{1}, \omega^{2}\right\}$. Agents' types are perfectly correlated, thus the two states of the world are $\omega=\left\{\left(\omega^{1}, \omega^{1}\right),\left(\omega^{2}, \omega^{2}\right)\right\}$; in addition, we take $\operatorname{prob}\left(\omega^{1}, \omega^{1}\right)=\operatorname{prob}\left(\omega^{2}, \omega^{2}\right)=0.5$.

We construct the example so that no player has state-dependent preferences and assuming that it is a strictly dominant strategy for each type of each agent to participate with $P 1$. The corresponding payoffs are represented in the table below, in which the first payoff is that of $P 1$, who chooses the row, the second payoff is that of $P 2$, who chooses the column, and the last two payoffs are those of $A 1$ and A2, given that they participate with $P 1$.

\begin{tabular}{|c|c|c|c|c|c|}
\hline & \multicolumn{5}{|c|}{$Y N$} \\
\hline \multirow{5}{*}{$Y N$} & & $x_{21}$ & $x_{22}$ & $x_{23}$ & $x_{24}$ \\
\hline & $x_{11}$ & $(\eta, 0,-1,-1)$ & $(\eta, 0,0,0)$ & $(\eta, 0,-1,-1)$ & $(1,0,9,6)$ \\
\hline & $x_{12}$ & $(\eta, 0,7,0)$ & $(\eta, 0,-1,-1)$ & $(2,0,6,9)$ & $(\eta, 0,-1,-1)$ \\
\hline & $x_{13}$ & $(\eta, 0,-1,-1)$ & $(2,0,6,9)$ & $(\eta, 0,-1,-1)$ & $(\eta, 0,7,0)$ \\
\hline & $x_{14}$ & $(1,0,9,6)$ & $(\eta, 0,-1,-1)$ & $(\eta, 0,0,0)$ & $(\eta, 0,-1,-1)$ \\
\hline
\end{tabular}

Table 1: Players' payoffs in state $\left(\omega^{1}, \omega^{1}\right)$ and $\left(\omega^{2}, \omega^{2}\right)$

We also let $\eta<0$ be an arbitrary large loss for P1.

Principals' decisions can be made contingent on the messages they receive from both agents. We let $\gamma_{j}: M_{j} \rightarrow \Delta\left(X_{j}\right)$ be a decision rule for principal $j$, holding fixed the agents' participation choices of dealing with $P 1$ only. With some abuse of language, we refer to $\gamma_{j}$ as to a mechanism for principal $j=1,2$. To simplify exposition, we let $M_{j}^{i}=\Omega^{i}$ for every $j$ and $i$. We fix P2's mechanism to be

$$
\tilde{\gamma}_{2}\left(m_{2}^{1}, m_{2}^{2}\right)= \begin{cases}x_{24} & \text { if he receives the message } \omega^{1} \text { from both } A 1 \text { and A2 } \\ x_{23} & \text { if he receives } \omega^{1} \text { from A1 and } \omega^{2} \text { from A2 } \\ x_{22} & \text { if he receives } \omega^{2} \text { from A1 and } \omega^{1} \text { from A2 } \\ x_{21} & \text { if he receives the message } \omega^{2} \text { from both A1 and A2. }\end{cases}
$$


Along the lines of Lemma 1, we let P1 post the mechanism

$$
\gamma_{1}^{\prime}\left(m_{1}^{1}, m_{1}^{2}\right)= \begin{cases}x_{11} & \text { if he receives the message } \omega^{1} \text { from both A1 and A2 } \\ x_{12} & \text { if he receives } \omega^{1} \text { from } A 1 \text { and } \omega^{2} \text { from A2 } \\ x_{13} & \text { if he receives } \omega^{2} \text { from } A 1 \text { and } \omega^{1} \text { from A2 } \\ x_{14} & \text { if he receives the message } \omega^{2} \text { from both A1 and A2. }\end{cases}
$$

Given $\left(\gamma_{1}^{\prime}, \tilde{\gamma}_{2}\right)$, we first show that the agents' message game exhibits a unique equilibrium, in which each type of each agent is untruthful to $P 1$ with a strictly positive probability: this yields $P 1$ a payoff of $3 / 2$. In fact, $\left(\gamma_{1}^{\prime}, \tilde{\gamma}_{2}\right)$ induce the game reported in Table 2.

\begin{tabular}{|c|cccc|}
\hline & $\left(\omega^{1}, \omega^{1}\right)$ & $\left(\omega^{1}, \omega^{2}\right)$ & $\left(\omega^{2}, \omega^{1}\right)$ & $\left(\omega^{2}, \omega^{2}\right)$ \\
\hline$\left(\omega^{1}, \omega^{1}\right)$ & $(9,6)$ & $(-1,-1)$ & $(-1,-1)$ & $(6,9)$ \\
$\left(\omega^{1}, \omega^{2}\right)$ & $(0,0)$ & $(-1,-1)$ & $(-1,-1)$ & $(7,0)$ \\
$\left(\omega^{2}, \omega^{1}\right)$ & $(7,0)$ & $(-1,-1)$ & $(-1,-1)$ & $(0,0)$ \\
$\left(\omega^{2}, \omega^{2}\right)$ & $(6,9)$ & $(-1,-1)$ & $(-1,-1)$ & $(9,6)$ \\
\hline
\end{tabular}

Table 2: Agents' payoffs in the game induced by $\left(\gamma_{1}^{\prime}, \tilde{\gamma}_{2}\right)$.

In Table 2, A1 chooses the rows and A2 chooses the columns. Each agent reports a type $\omega \in$ $\left\{\omega^{1}, \omega^{2}\right\}$ to $P 1$ and $P 2$, respectively. Observe that, for A2, the strategies $\left(\omega^{1}, \omega^{2}\right)$ and $\left(\omega^{2}, \omega^{1}\right)$ are strictly dominated by $\left(\omega^{1}, \omega^{1}\right)$. Upon eliminating them, one can check that reporting $\left(\omega^{1}, \omega^{2}\right)$ is strictly dominated by $\left(\omega^{2}, \omega^{2}\right)$, and that $\left(\omega^{2}, \omega^{1}\right)$ is strictly dominated by $\left(\omega^{1}, \omega^{1}\right)$ for A1. Iterated elimination of these strictly dominated strategies yields the reduced game in Table 3.

\begin{tabular}{|c|cc|}
\hline & $\left(\omega^{1}, \omega^{1}\right)$ & $\left(\omega^{2}, \omega^{2}\right)$ \\
\hline$\left(\omega^{1}, \omega^{1}\right)$ & $(9,6)$ & $(6,9)$ \\
$\left(\omega^{2}, \omega^{2}\right)$ & $(6,9)$ & $(9,6)$ \\
\hline
\end{tabular}

Table 3: The reduced agents' game induced by $\left(\gamma_{1}^{\prime}, \tilde{\gamma}_{2}\right)$.

The game in Table 3 has no pure strategy equilibrium: the unique mixed-strategy Nash equilibrium prescribes that both $A 1$ and A2 randomize between $\left(\omega^{1}, \omega^{1}\right)$ and $\left(\omega^{2}, \omega^{2}\right)$ with equal probabilities. This equilibrium yields an expected payoff of $3 / 2$ to $P 1$, which is sustained by the array of mechanisms $\left(\gamma_{1}^{\prime}, \tilde{\gamma}_{2}\right)$ and the agents' (fully mixed) communication behavior.

We next show that, given $\tilde{\gamma}_{2}$, there is no way for P 1 to obtain a payoff of $3 / 2$ by means of a direct mechanism $\tilde{\gamma}_{1}^{\prime}$ which induces each type of each agent to be truthful to him. Hence, Lemma 1 does not hold.

To establish the result, we first consider the case in which $\tilde{\gamma}_{1}^{\prime}$ is a deterministic direct mechanism. To yield P1 a payoff of $3 / 2$, the mechanisms $\left(\tilde{\gamma}_{1}^{\prime}, \tilde{\gamma}_{2}\right)$ must induce an equilibrium in the agents' game such that when $\left(\omega^{i}, \omega^{i}\right)$ realizes, with $i=1,2$, either the decisions $\left(x_{12}, x_{23}\right)$ or $\left(x_{13}, x_{22}\right)$ 
are played with positive probability. Suppose that $\left(\omega^{1}, \omega^{1}\right)$ realizes. In this case, for $\left(x_{12}, x_{23}\right)$ to arise in an equilibrium in which agents are truthful to $P 1$, it must be $\tilde{\gamma}_{1}^{\prime}\left(\omega^{1}, \omega^{1}\right)=\left\{x_{12}\right\}$. In the corresponding agents' game, type $\omega^{1}$ must report $\left(\omega^{1}, \omega^{1}\right)$, and type $\omega^{2}\left(\omega^{1}, \omega^{2}\right)$ for $\left\{x_{12}, x_{23}\right\}$ to be played with positive probability in a truth-telling equilibrium. This point is illustrated in Table 4 which only considers the agents' payoffs that can be supported in a truth-telling equilibrium.

\begin{tabular}{|c|cccc|}
\hline & $\left(\omega^{1}, \omega^{1}\right)$ & $\left(\omega^{1}, \omega^{2}\right)$ & $\left(\omega^{2}, \omega^{1}\right)$ & $\left(\omega^{2}, \omega^{2}\right)$ \\
\hline$\left(\omega^{1}, \omega^{1}\right)$ & $(-1,-1)$ & $(6,9)$ & $(.,)$. & $(.,)$. \\
$\left(\omega^{1}, \omega^{2}\right)$ & $(-1,-1)$ & $(7,0)$ & $(.,)$. & $(.,)$. \\
$\left(\omega^{2}, \omega^{1}\right)$ & $(.,)$. & $(.,)$. & $(.,)$. & $(.,)$. \\
$\left(\omega^{2}, \omega^{2}\right)$ & $(.,)$. & $(.,)$. & $(.,)$. & $(.,)$. \\
\hline
\end{tabular}

Table 4: Agents' payoffs in the game induced by $\tilde{\gamma}_{1}^{\prime}$ such that $\tilde{\gamma}_{1}^{\prime}\left(\omega^{1}, \omega^{1}\right)=\left\{x_{12}\right\}$.

Clearly, when A2 chooses $\left(\omega^{1}, \omega^{2}\right)$, A1 prefers $\left(\omega^{1}, \omega^{2}\right)$, which makes impossible to implement the decisions $\left\{x_{12}, x_{23}\right\}$ at equilibrium. A similar reasoning guarantees that, if $\tilde{\gamma}_{1}^{\prime}\left(\omega^{1}, \omega^{1}\right)=\left\{x_{13}\right\}$, $\left(\left(\omega^{1}, \omega^{2}\right),\left(\omega^{1}, \omega^{1}\right)\right)$ cannot be supported in a truth-telling equilibrium, since A1 prefers $\left(\omega^{1}, \omega^{1}\right)$, which makes impossible to implement $\left\{x_{13}, x_{22}\right\}$ at equilibrium. Given $\tilde{\gamma}_{2}$, a similar reasoning applies if $\left(\omega^{2}, \omega^{2}\right)$ realizes.

We next consider the case in which $\tilde{\gamma}_{1}^{\prime}$ is a stochastic direct mechanisms. Then, given $\tilde{\gamma}_{2}$, every such lottery would put a positive weight on $\eta$, whose value can be set arbitrarily small to make impossible for $\tilde{\gamma}_{1}^{\prime}$ to yield $3 / 2$ to $P 1$.

The result suggests that Assumption E is tight to establish Lemma 1 and, hence, Proposition 1. The possibility for agents to communicate with $P 2$ when participating with $P 1$ constitutes a source of moral hazard from the viewpoint of $P 1$. Assumption E severely limits this effect by guaranteeing that, given participation, $P 1$ is perfectly informed of the messages received by his opponent. The example also suggests that, when Assumption E is dropped, in order for $P 1$ to be able to reconstruct the decisions induced by an arbitrary mechanism $\gamma_{1}^{\prime}$ one should consider direct mechanism that allow him to privately communicate with agents, as in the single-principal setting of Myerson (1982). ${ }^{23}$

\section{Conclusion}

This paper studies the role of direct mechanisms in games in which principals compete for the exclusive right to serve agents. We establish two main results. First, restricting principals to use such mechanisms may involve a loss of generality. Second, when principals post direct mechanisms, we identify a set of equilibrium outcomes that survive against unilateral deviations towards arbitrary mechanisms. This rationalizes the approach followed in applications.

\footnotetext{
${ }^{23}$ A first example in this direction is constructed in Attar et al. (2012).
} 
Our analysis could be extended to cope with situations in which principals can take some actions after the mechanisms have been posted. This possibility arises in economic contexts in which contract renegotiation is an issue. In the specific case of insurance markets with private information, following the original intuitions of Wilson (1977) and Riley (1979), several authors have investigated the implications of insurers withdrawing and/or adding contracts after the initial negotiation stage. ${ }^{24}$ In terms of our model, this feature can be captured by assuming that the mechanism offered by principal $j$ does not uniquely determine his decision, for a given array of agents' participation and messages. That is, a principal can take an additional decision after messages are sent. Identifying the role of direct mechanisms in competitive models of contract renegotiation is an open issue for future research. ${ }^{25}$

\section{References}

Asheim, G. B. And T. Nilssen (1996): "Non-discriminating renegotiation in a competitive insurance market," European Economic Review, 40, 1717-1736.

Attar, A., E. Campioni, And G. Piaser (2015): "On Competing Mechanisms under Exclusive Competition," TSE-Working Paper 15-609.

Attar, A., E. Campioni, G. Piaser, And U. Rajan (2012): "Competing mechanism games of moral hazard: communication and robustness," Review of Economic Design, 16, 283-296.

Bernheim, B. D. And M. D. Whinston (1998): "Exclusive Dealing," Journal of Political Economy, 106, 64-103.

Bisin, A. And P. Gottardi (2006): "Efficient Competitive Equilibria with Adverse Selection," Journal of Political Economy, 114, 485-516.

Burguet, R. And J. Sakovics (1999): "Imperfect competition in auction designs," International Economic Review, 40, 231-247.

Caillaud, B., B. Jullien, And P. Picard (1995): "Competing vertical structures: precommitment and renegotiation," Econometrica, 621-646.

Eeckhout, J. And P. Kircher (2010): "Sorting versus screening: Search frictions and competing mechanisms," Journal of Economic Theory, 145, 1354-1385.

Epstein, L. G. And M. Peters (1999): "A revelation principle for competing mechanisms," Journal of Economic Theory, 88, 119-160.

\footnotetext{
${ }^{24}$ See, for instance, Hellwig (1987), Asheim and Nilssen (1996) and Netzer and Scheuer (2010).

${ }^{25}$ In this respect, an important starting point is provided by Szentes (2010) who shows that communication is instrumental to establish a folk theorem for games of complete information in which principals can take actions after having posted their mechanisms.
} 
GAL-OR, E. (1991): "A common agency with incomplete information," RAND Journal of Economics, 22, 274-286.

GAL-OR, E. (1997): "Multiprincipal agency relationships as implied by product market competition," Journal of Economics \& Management Strategy, 6, 235-256.

Gottardi, P. And S. Auster (2016): "Competing Mechanisms in Markets for Lemons," in 2016 Meeting Papers, Society for Economic Dynamics, 264.

Guerrieri, V., R. Shimer, And R. Wright (2010): "Adverse selection in competitive search equilibrium," Econometrica, 78, 1823-1862.

HAN, S. (2007): "Strongly robust equilibrium and competing-mechanism games," Journal of Economic Theory, 137, 610-626.

- (2015): "Robust Competitive Auctions," Economics Letters, 136C, 207-210.

Hellwig, M. (1987): "Some recent developments in the theory of competition in markets with adverse selection ," European Economic Review, 31, 319-325.

Inderst, R. AND A. WAMBACH (2002): "Capacity constrained firms in (labor) markets with adverse selection," Economic Theory, 19, 525-548.

MARTimort, D. (1996): "Exclusive dealing, common agency and multiprincipal incentives theory," RAND Journal of Economics, 27, 1-31.

Martimort, D. And S. Piccolo (2010): "The Strategic Value of Quantity Forcing Contracts," American Economic Journal: Microeconomics, 2, 204-229.

Martimort, D. And L. A. Stole (2002): "The revelation and delegation principles in common agency games," Econometrica, 70, 1659-1673.

McAfee, P. (1993): "Mechanism design by competing sellers," Econometrica, 61, 1281-1312.

Myerson, R. B. (1982): "Optimal coordination mechanisms in generalized principal-agent problems," Journal of Mathematical Economics, 10, 67-81.

Netzer, N. And F. Scheuer (2010): "Competitive Markets without Commitment," Journal of Political Economy, 118, 1079 - 1109.

O’Brien, D. P. And G. Shaffer (1997): "Nonlinear Supply Contracts, Exclusive Dealing, and Equilibrium Market Foreclosure," Journal of Economics $\&$ Management Strategy, 6, 755-785.

Pagnozzi, M. And S. Piccolo (2013): "Information sharing between vertical hierarchies," Games and Economic Behavior, 79, 201-222. 
PECK, J. (1997): "A note on competing mechanisms and the revelation principle," Mimeo, Ohio State University.

(2015): "Sky-lift Pricing with Imperfect Competition: An exercise in Competing Mechanisms," Mimeo, Ohio State University.

Peters, M. (1997): "A competitive distribution of auctions," Review of Economic Studies, 64, $97-123$.

(2001): "Common Agency and the Revelation Principle," Econometrica, 69, 1349-1372.

- (2003): "Negotiation and take-it or leave-it in common agency," Journal of Economic Theory, 111, 88-109.

(2004): "Pure strategy and no-externalities with multiple agents," Economic Theory, 23, $183-194$.

- (2014): "Survey on Competing Mechanisms 2 - The revelation principle," Canadian Journal of Economics, 47(2), 373-397.

Peters, M. And S. Severinov (1997): "Competition among sellers who offer auctions instead of prices," Journal of Economic Theory, 75, 141-179.

Prescott, E. And R. Townsend (1984): "Pareto Optima and Competitive Equilibria with Adverse Selection and Moral Hazard," Econometrica, 52, 21-45.

Rey, P. And T. Vergé (2008): "Economics of Vertical Restraints," in Handbook of Antitrust Economics, MIT Press, 353-390.

RiLey, J. G. (1979): "Informational Equilibrium," Econometrica, 47, 331-59.

Rochet, J.-C. (1985): "The taxation principle and multi-time Hamilton-Jacobi equations," Journal of Mathematical Economics, 14, 113-128.

Rothschild, M. And J. Stiglitz (1976): "Equilibrium in Competitive Insurance Markets," Quarterly Journal of Economics, 90, 629-649.

Schlee, E. And H. Chade (2012): "Optimal insurance with adverse selection," Theoretical Economics, 7.

Stiglitz, J. E. (1977): "Monopoly, Non-linear Pricing and Imperfect Information: The Insurance Market," Review of Economic Studies, 44, 407-430.

Szentes, B. (2010): "A note on Mechanism games with multiple principals and three or more agents by T. Yamashita," Mimeo, London School of Economics. 
VIRÀG, G. (2010): "Competing Auctions: Finite Markets and Convergence," Theoretical Economics, 5, 241-274.

Wilson, C. (1977): "A model of insurance markets with incomplete information," Journal of Economic Theory, 16, 167-207.

YAmashita, T. (2010): "Mechanism games with multiple principals and three or more agents," Econometrica, 78, 791-801. 